\title{
EFICÁCIA DA LASERTERAPIA NA REDUÇÃO DE MORBIDADE APÓS CIRURGIA DE TERCEIROS MOLARES - UMA REVISÃO DE LITERATURA
}

\author{
LASERTHERAPY EFFECTIVENESS IN REDUCING MORBIDITY AFTER 3 \\ MOLAR SUGERY - A REVIEW OF LITERATURE
}

Maria Helena Chaves de Vasconcelos Catão

Aline Monteiro de Moura

Armiliana Soares Nascimento
Doutora em Odontologia (Laser na Odontologia) pela Universidade Federal da Bahia. Professora; Doutora do Programa de Pós-Graduação em Odontologia da UEPB. Professora titular da Disciplina de Dentística da Universidade Estadual da Paraíba/ UEPB, Campina Grande - PB, Brasil.

Cirurgiã-dentista, Campina Grande - PB, Brasil.

Mestranda do Programa de Pós-Graduação em Odontologia pela Universidade Estadual da Paraíba/ UEPB, Campina Grande - PB, Brasil.

\section{Resumo}

Todo elemento dentário que, passado o seu período normal de erupção, não irrompe na cavidade oral, é classificado como retido. Os terceiros molares são os que mais frequentemente permanecem retidos, necessitando de tratamento cirúrgico, envolvendo, invariavelmente, trauma ao osso e tecidos moles da região. Isto acarreta maior chance de desenvolvimento de alterações pós-operatórias como a dor, o edema e o trismo, diminuindo, muitas vezes, a qualidade de vida do paciente e o afastando de suas atividades rotineiras. Assim, vários estudos têm sido concretizados com o intuito de diminuir a morbidade de tal procedimento e instituir terapias alternativas, dentre elas, a utilização do laser de baixa intensidade. O presente estudo objetivou realizar uma revisão de literatura sobre a utilização do laser de baixa potência na cirurgia bucomaxilofacial, abordando a eficácia do mesmo na redução da morbidade pós-operatória relacionada a edema, dor e trismo de pacientes submetidos a cirurgias dos terceiros molares. Para isso, foram feitas consultas nos bancos de dados disponíveis na internet, Lilacs, Bireme e Scielo. Como palavras-chave foram empregadas as seguintes: cirurgia, dentes inclusos, terceiros molares, laser de baixa potência, trismo, dor pós-operatória, laserterapia de baixa potência, cirurgia dentoalveolar, cicatrização de feridas cirúrgicas. Após a pesquisa bibliográfica, conclui-se que a maioria dos estudos sobre a aplicabilidade do laser de baixa potência na cirurgia bucomaxilofacial apresentou efeitos benéficos deste no controle da dor, edema e trismo pós-operatórios, inclusive diminuindo a quantidade de medicação necessária.

Palavras-chave: LASER DE BAIXA POTÊNCIA; TERCEIRO MOLAR; EXODONTIA; CIRURGIA BUCOMAXILOFACIAL.

\section{Abstract}

Every tooth after their normal period of eruption doesn't break out in the oral cavity is classified as held. The third molars are the most frequent are still retained, requiring surgical treatment, involving, invariably, trauma to the bone and soft tissues of the region. This results in a greater chance of developing changes post-operative complications such as pain, swelling and trismus, declined, many times, the patient's quality of life and the drift away from their daily activities. Thus, many studies have been carried out with the intention of reducing the morbidity of this procedure is to establish alternative therapies among them, the use of low intensity laser. The present study aimed to undertake a review of the literature on the use of low level laser in oral and maxillofacial surgery, addressing the efficacy of same in the reduction of post-operative morbidity related to edema, pain, and trismus of patients undergoing surgeries of third molars. For this reason was conducted consultations in the databases available on the internet, Lilacs, Bireme and Scielo. As key words were used the following: surgery, unerupted teeth, third molars, low level laser, trismus, postoperative pain, laser therapy of low power, dentoalveolar surgery, healing of surgical wounds. After the bibliographic research concludes that the majority of studies on the applicability of low level laser in maxillofacial surgery showed beneficial effects of this in the control of pain, swelling and trismus postoperative, including decreasing the amount of medication needed.

Keyword: LOW LEVEL LASER; THIRD MOLAR; DENTAL EXTRACTION; ORAL AND MAXILLOFACIAL SURGERY. 


\section{INTRODUÇÃO}

A remoção cirúrgica de terceiros molares normalmente envolve dor, edema e trismo no período pós-operatório. Os fatores que contribuem para essa situação geralmente têm origem no processo inflamatório, com início diante de um trauma cirúrgico, ${ }^{1}$ resultante basicamente da extensão da cirurgia, da manipulação dos tecidos moles e da resposta tecidual. Mesmo seguindo uma técnica cirúrgica adequada e uma manipulação tecidual cuidadosa, nunca se consegue bloquear totalmente a sintomatologia pós-operatória. ${ }^{2,3}$

Desse modo, conhecendo a capacidade do LASER de proporcionar melhor resposta inflamatória, com consequente redução do edema, minimização da sintomatologia dolorosa, e bioestimulação celular, a laserterapia apresenta-se como alternativa iminente em todo e qualquer processo que apresente reação inflamatória, dor e necessidade de regeneração tecidual. ${ }^{3}$ Este pode ser utilizado nas mais diversas especialidades, respeitando os princípios terapêuticos, efeitos produzidos no organismo e a metodologia correta de aplicação. ${ }^{4}$

A aplicação dos diferentes tipos de lasers possibilita grandes alterações nos procedimentos médicos e odontológicos, proporcionando redução do tempo cirúrgico, melhor recuperação dos pacientes das complicações pós-operatórias, redução de edema e, ainda, facilita a bioestimulação dos tecidos moles, além de permitir um maior controle e domínio das dores crônicas. ${ }^{5}$

Com isso, propusemos a realização de uma revisão de literatura sobre a utilização do laser de baixa potência na cirurgia bucomaxilofacial, abordando a eficácia do mesmo na redução da morbidade pós-operatória relacionada a edema, dor e trismo de pacientes submetidos a cirurgias dos terceiros molares.

\section{REVISÃo DE Literatura}

A luz, desde os primórdios da civilização, vem sendo utilizada com finalidades terapêuticas, contudo, só a partir dos princípios termodinâmicos de Einstein, em 1917, é que o físico Gordon Gold desenvolveu a tecnologia conhecida atualmente como laser. ${ }^{3}$

O laser é uma forma de radiação não ionizante, altamente concentrada, que em contato com os diferentes tecidos resulta, de acordo com o tipo, efeitos térmicos, fotoquímicos e não lineares. ${ }^{6}$

Ao contrário de outras formas de radiação usadas terapeuticamente, como os raios $\mathrm{X}$, raios gama e nêutrons, a irradiação laser não é invasiva e é bem tolerada pelos tecidos. Por isso, acredita-se que esta seja uma excelente opção de tratamento, já que apresenta efeitos benéficos para os tecidos irradiados, como a ativação da microcirculação, produção de novos capilares, efeitos anti-inflamatórios e analgésicos, além de estimular o crescimento e a regeneração celular. ${ }^{7}$

O feixe de luz laser ao incidir sobre o tecido pode desencadear quatro tipos de interação: a) Absorção - A luz é absorvida por componentes dos tecidos como a água, hemoglobina ou melanina, e é transformada em outras formas de energia (calórica, química etc.), atuando no interior dos tecidos onde foi absorvida e também propagando seus efeitos para os tecidos vizinhos. b) Reflexão - Parte da luz incidente é refletida e perdida. c) Difusão ou espalhamento - Parte da luz se espalha pelos tecidos, perdendo sua potência. d) Transmissão - A luz atravessa toda a espessura do tecido. ${ }^{8}$

Assim, os efeitos biológicos sobre os tecidos são dependentes do comprimento de onda e da densidade energética das radiações, destacando os fototérmicos (fotocoagulação, fotoablação e fotovaporização) e os fenômenos fotoquímicos. ${ }^{6,8}$

Os fenômenos fototérmicos são característicos dos lasers cirúrgicos e podem determinar a desnaturação do conteúdo proteico celular, coagulando, vaporizando ou ablacionando o conteúdo hídrico das células e carbonizando os tecidos, enquanto os fenômenos fotoquímicos, agindo sob os receptores da membrana celular, promovem a absorção de fótons por biomoléculas intracelulares produzindo a estimulação ou inibição de atividades enzimáticas e de reações fotoquímicas, permitindo a instalação de processos fisiológicos de natureza terapêutica, como ações analgésicas, anti-inflamatórias e de bioestimulação tecidual. ${ }^{6,8}$

Essa radiação vem sendo empregada em procedimentos operatórios com o objetivo de aumentar os benefícios cirúrgicos, melhorando o prognóstico clínico, tendo como principais vantagens a desinfecção do campo operatório, ausência de vibração, vaporização das lesões, conforto para o paciente, propriedades anti-inflamatórias e bioestimuladoras, ${ }^{9,10}$ precisão na destruição tecidual, mínimo dano aos tecidos adjacentes, efeito hemostático, redução da dor e do edema, esterilização do campo cirúrgico e possibilidade de controle microscópico e endoscópio. ${ }^{11}$

De acordo com a potência de emissão, pode ser classificada em: laser de alta, de média e de baixa intensidade. ${ }^{6,8}$ 
Os lasers de baixa intensidade são radiações emitidas sem potencial destrutivo, sendo também denominados de soft-laser, laser mole, laser frio ou laser terapêutico. Surgiram com Mester, na Hungria, em 1967, e têm sido utilizados pelos cirurgiões-dentistas brasileiros há cerca de quinze anos nas mais variadas áreas da odontologia, procurando investigar a viabilização do seu emprego como modalidade de tratamento de diferentes afecções do complexo maxilofacial.12

Entre as áreas odontológicas empregadas, tem-se a cirurgia oral, a periodontia, a dentística, a ortodontia, a pediatria, a endodontia, a estomatologia, entre outras. ${ }^{13}$

$\mathrm{Na}$ cirurgia, mais precisamente a exodontia de terceiros molares que envolve, invariavelmente, trauma ao osso e tecidos moles, ${ }^{1,3}$ o laser tem se mostrado uma excelente opção de tratamento pós-operatório.

A remoção de dentes impactados é um dos procedimentos cirúrgicos mais realizados pelos cirurgiões bucomaxilofaciais, tendo como indicações principais a prevenção da cárie dentária, da doença periodontal, da pericoronarite, da reabsorção de raízes de dentes adjacentes, de cistos e tumores odontogênicos, de fraturas da mandíbula, bem como por indicação ortodôntica ou protética. ${ }^{14}$

De acordo com o tipo de cirurgia, técnica utilizada, resposta do paciente e apesar da utilização de medicamentos, a sintomatologia dolorosa no pós-operatório pode ser variável, assim como o edema. Além disso, o desconforto geral no paciente pode limitar a realização de suas atividades diárias, além de dificultar uma adequada higienização bucal pós-cirúrgica, retardando a cicatrização. ${ }^{15}$

Para amenizar esse processo, a terapia medicamentosa associada a medidas locais de controle pós-operatório, como a crioterapia, repouso muscular, entre outras, vem sendo empregada, destacando o uso de medicamentos, como os anti-inflamatórios não-esteroidais (AINES) e os corticosteroides. ${ }^{16}$

O laser de baixa intensidade (LBI) constitui opção terapêutica que vem recebendo atenção de muitos estudos no processo de garantir conforto ao paciente. ${ }^{17}$

No trabalho realizado por Viegas et al.,$^{18}$ usando o laser de baixa intensidade de $\mathrm{He}-\mathrm{Ne}$ para o tratamento de edema e trismo acentuados, após a exodontia do terceiro molar inferior esquerdo puderam observar uma redução do edema e do trismo cerca de $70 \%$, após oito horas da primeira aplicação.

Também Maluf et al. ${ }^{3}$ relataram resultados satisfatórios no controle da dor e edema, em um caso de pós-operatório de extração de um terceiro molar semi-incluso com retenção distal, com uma única aplicação de laser associado ao uso de anti-inflamatório não esteroidal, imediatamente antes da cirurgia.

Laureano-Filho et al., ${ }^{1}$ avaliando a influência do LBI na redução da dor, edema e trismo, em cirurgias de terceiros molares, com aplicações feitas no pré-operatório, no pós-operatório imediato e em 24 e 48 horas após, mostraram efetividade do laser na diminuição da dor e do trismo, porém sem eficácia sobre o edema, durante o período estudado.

A associação do LBI com anti-inflamatórios também foi descrita por Markovic e Todorovic, ${ }^{17}$ onde avaliaram a eficácia da laserterapia e da dexametasona para minimizar o edema após cirurgia de terceiro molar. Desse estudo concluíram que a combinação do laser com o corticoide intramuscular foi mais eficiente na redução do edema.

\section{Discussão}

Atualmente, o Brasil dispõe de aparelhos de laser de baixa intensidade e alguns profissionais já o utilizam como mais uma ferramenta de tratamento coadjuvante para melhorar a qualidade de vida de seus pacientes, em seus consultórios. Porém, ao adquirir o aparelho de laser, é importante que o profissional conheça as características, os efeitos de cada sistema e sua forma de aplicação.

Segundo Almeida-Lopes e Massini, ${ }^{19}$ Conlan et al. ${ }^{20}$ Lopes e Eduardo, ${ }^{21}$ Catão et al. $, 22,23$ os lasers de baixa potência apresentam efeitos analgésicos (estimulam a liberação de endorfinas, inibem sinais nociceptores e reduzem sintomatologia dolorosa), são anti-inflamatórios (reduzem o edema e a hiperemia) e têm ação bioestimulante (aceleram a cicatrização de feridas, estimulam a remodelação e o reparo ósseos, restauram a função neural após injúrias e modulam as células do sistema imune para favorecer o reparo).

A biomodulação ou a bioestimulação é, sem dúvida, uma das áreas de maior controvérsia no uso de lasers em odontologia, ${ }^{6}$ entretanto, observa-se que alguns estudos vieram demonstrar que a laserterapia não cirúrgica possui este tipo de atividade, sendo sugerido que sejam realizadas novas avaliações ou continuação destes estudos para a sua correta comprovação. ${ }^{24,25}$

Pelas suas propriedades, o LBI vem sendo usado para complementar a ação anti-inflamatória de alguns fármacos, além de acelerar a reparação tecidual em cirurgias de terceiros molares.,17,18 Este tem sido usado como alternativa terapêutica 
para coadjuvar a ação de fármacos no controle da dor, edema e outras manifestações pós-operatórias indesejáveis das cirurgias de terceiros molares. Seus efeitos analgésico, anti-inflamatório e de biomodulação tecidual, são referidos em vários trabalhos. $3,8,17,18$

Vários relatos na literatura têm mostrado resultados satisfatórios com relação à diminuição da dor, edema e trismo pós-operatórios, além da aceleração da cicatrização de retalhos cirúrgicos após exodontias com a aplicação do laser de baixa intensidade,${ }^{26}$ bem como a regeneração neural, em lesões do nervo alveolar inferior. ${ }^{27}$

\section{Considerações finais}

A laserterapia aplicada à cirurgia de terceiros molares é descrita na literatura, porém a avaliação da dor, edema e trismo têm sido feitos após utilização de diferentes protocolos de irradiação. Sugere-se assim a realização de mais estudos para se poder validar cientificamente as questões ainda empíricas acerca do uso do laser de baixa intensidade na redução da morbidade pós-cirúrgica de terceiros molares, bem como elucidar uma dose ótima para cada caso, aumentando cada vez mais a credibilidade deste tipo de terapia na clínica de cirurgia bucomaxilofacial.

\section{REFERÊNCIAS BIBLIOGRÁFICAS}

1. Laureano-filho JR, Camargo IB, Firmo ACB, Silva, EDO. A influência do laser de baixa intensidade na redução de edema, dor e trismo no pós-operatório de cirurgia de terceiros molares inferiores inclusos: resultado preliminar com 13 casos. Rev Cirur Traumatol Buco-Maxilo-Facial, 2008 jun. 8(1): 47-56.

2. Capuzzi P., Monyebugnoli L., Vaccaro MA. Extraction of impacted third molars. A longitudinal prospective study on factors that affect postoperative recovery. Oral Surgery, Oral Med, Oral Pathol, Oral Radiol and Endodont. 1997; 77: 341-3.

3. Maluf Al, Ughini GC, Maluf RP, Pagnoncelli RM. Utilização de Laser Terapêutico em Exodontia de Terceiros Molares Inferiores. Rev Gaúcha Odontol, Porto Alegre. 2006, abr./ jun. 54(2): 182-184.

4. Brugnera JRA, Pinheiro ALB. Laser em Odontologia moderna. São Paulo: Ed Pancast. 1997.

5. Damante CA. Avaliação clínica e histológica dos efeitos de laser em baixa intensidade (Ga-
AlAs) na cicatrização após gengivoplastia em humanos. Dissertação de Mestrado em Odontologia. Faculdade de Odontologia de Bauru - Bauru. 2003. 92.

6. Brugnera JRA, Pinheiro ALB. Laser na Odontologia Moderna. São Paulo; Pancast, 2002.

7. Pesevska S., Nakova M., Pejcic A., Ivanovski K., Angelov N., Mindova S. Biostimulative Laser Therapy: base for favorized and accented results in Dentistry. Acta Fac Med Naiss. 2006; 23(1): 75-78.

8. Genovese WJ. Laser de baixa intensidade. Aplicações terapêuticas em odontologia. São Paulo: Santos, 2007; 130.

9. Pozza DH, Mello Eda, Blaya Ds, Pinheiro Alb, Oliveira Mg. Análise comparativa entre os tempos gastos na apicetomia por ponta diamantada e pelos lasers co2 e er:yag na apicectomia. Rev Clín Pesqu Odont. 2005; 2(2): 97-102.

10. Wilder-smith P., Dang J., Kurosaki T. Investigating the range of surgical effects on soft tissue produced by a carbon dioxide laser. J Am Dental Assoc. 1997; 28(5): 583-588.

11. Fisher SE, Frame JW. The effects of the carbon dioxide surgical laser on oral tissues. $\mathbf{J}$ Oral Maxillofacial Surgery. 1984; 22(6): 414-425.

12. Oliveira JAGP, Loyola AM, Costa IM, Garcia VG, Alves GC. Ação da irradiação laser (arsênio-gálio) sobre a cronologia do processo de reparo em feridas cutâneas - estudo histológico em ratos. Rev Odont Brasil Central. 1997; 6(21): 28-31.

13. Gutknecht N., Eduardo CP. A odontologia e o laser: atuação na especialidade odontológica. São Paulo: Quintessence. 2003.

14. Neills GM, Peterson LJ. Impacted teeth. In: MILORO, M. Peterson's principles of oral and maxillofacial surgery. 2. ed. São Paulo: Santos, 2008; 139-56.

15. Ferreira LS, Meneguzzo DT, Freitas PM. Fototerapia com laser em baixa intensidade na modulação dos sintomas do pós-operatório cirúrgico. $26^{\circ}$. CIOSP - Atualização Clínica em Odontologia: Clínica do Dia-a-Dia, Editora Artes Médicas. São Paulo, 2008; 563-573.

16. Freitas JR. Terapêutica odontológica. Rio de Janeiro: Pedro Pinheiro. 1997.

17. Markovic AB, Todorovic L. Effectiveness of dexamethasone and low-power laser in minimizing oedema after third molar surgery: a clinical trial. Inter J Oral Maxillofacial Surgery. 2007; 36: 226-29. 
18. Viegas VN, Prietto L, Mezzomo LAM, Abreu MER, Pagnoncelli RM. Controle do edema: terapia medicamentosa $\mathrm{x}$ uso do laser. Rev ABO Nac. 2005, ago./set. 13(4): 245-50.

19. Almeida-Lopes L., Massini RJ. Laser e suas aplicações. São Paulo: DMC Equipamentos, 2000. 31.

20. Conlan MJ, Rapley JW, Cobb CM. Biostimulation of would healing by low-energy laser irradiation. J Clin Periodontol. 1996; 23: 492-96.

21. Lopes LM, Eduardo CP. Laserterapia em periodontia. In: Compêndio terapêutico periodontal. São Paulo: Artes Médicas. 1999; 293-307.

22. Catão MHCV, Gerby MEM, Gonçalves RC et al. A Laserterapia no tratamento da radiomucosite em paciente com carcinoma espinocelular no palato mole: relato de caso. Laser Dental Show, 2003; 8-9.

23. Catão MHCV. Os benefícios do laser de baixa intensidade na clínica odontológica na esto- matologia. Rev Bras Pesqu Odontol 2004 out./nov./dez.

24. Saito S, Shimizu N. Stimulatory effects of low power laser irradiation on bone regeneration in midpalatal suture during expansion in the rat. Am. J. Orthod. Dentofacial Orthop. 1997 mai. 111(5): 525-32.

25. Wilden L, Karthein R. Import of radiation phenomena of electrons and therapeutic lowlevel laser in regard to the mitochondrial energy transfer. J Clin Laser Med Surgery. 1998 jun. 16(3): 159-65.

26. Neiburger EJ. Rapid healing of gingival incisions by the helium-neon diode laser. J Mass Dent Soc. 1999; 48(1): 8-13.

27. Ozen T., Orhan K., Gorur I., Ozturk A. Efficacy of low level laser therapy on neurosensory recover after injury to the inferior alveolar nerve. Head Face Med. 2006; 2(1): 1-9.

Submetido em: 10/5/2012

Aceito em: 2/8/2012 